\title{
Transcriptome Classification Reveals Molecular Subgroups in Patients with Hepatitis B Virus
}

\author{
Conghui Zhang $\mathbb{D},{ }^{1}$ Jie Li $\mathbb{D},{ }^{1}$ Lan Yang $\mathbb{D},{ }^{2}$ Fengxia Xu $\mathbb{D},{ }^{2}$ Huiyuan She $\mathbb{D},{ }^{3}$ \\ and Xinghui Liu ${ }^{2}$ \\ ${ }^{1}$ Postgraduate Training Base in Shanghai Gongli Hospital, Ningxia Medical University, Pudong New Area, Shanghai 200135, China \\ ${ }^{2}$ Department of Clinical Laboratory, Shanghai Gongli Hospital, The Second Military Medical University, Pudong New Area, \\ Shanghai 200135, China \\ ${ }^{3}$ Department of Infectious Medicine, Shanghai Gongli Hospital, The Second Military Medical University, Pudong New Area, \\ Shanghai 200135, China
}

Correspondence should be addressed to Huiyuan She; 18930872968@163.com and Xinghui Liu; syliuxh@163.com

Conghui Zhang and Jie Li contributed equally to this work.

Received 6 January 2021; Revised 27 February 2021; Accepted 9 March 2021; Published 31 March 2021

Academic Editor: Tao Huang

Copyright $\odot 2021$ Conghui Zhang et al. This is an open access article distributed under the Creative Commons Attribution License, which permits unrestricted use, distribution, and reproduction in any medium, provided the original work is properly cited.

Hepatitis B virus (HBV) specifically infects hepatocytes, which can cause progressive liver fibrosis and a significantly increased risk of liver cancer. Multiple studies indicated host genetic, virological, and immunological factors could affect the HBV infection. However, the underlying mechanism involved in HBV infection remained unclear. Based on the analysis of gene expression data of $124 \mathrm{HBV}$ patients (GEO accession: GSE84044), molecular subgroups of patients infected with hepatitis B virus were identified in this study, including C1, C2, and C3 groups. The age, fiber, degree of chemical and inflammation, and gene expression difference were also compared among the three sampling groups. Furthermore, the liver index was calculated using 93 liver-specific genes. The liver-specific gene expression in different molecular subgroups of HBV patients was thoroughly analyzed and then was compared with fibrosis and inflammation levels. Results showed that the $\mathrm{C} 2$ group was the youngest and the C3 group had the highest degree of fibrosis and inflammation. Enrichment analysis showed that metabolism-related pathways were mainly expressed in the $\mathrm{Cl}$ and $\mathrm{C} 2$ groups, and inflammation-related pathways and proteoglycans in cancer were highly expressed in the C1 and C3 groups. The liver index was higher in the $\mathrm{C} 2$ group than in the $\mathrm{C} 1$ and $\mathrm{C} 3$ groups, and it was the lowest in the C3 group. Macrophage M1/M2 and neutrophils were significantly different in the three groups. M1 was mainly abundant in the C3 group, and M2 and neutrophils were mainly abundant in the $\mathrm{C} 2$ group. This study provides novel information to understand the mechanisms of HBV infection in chronic hepatitis $\mathrm{B}(\mathrm{CHB})$ patients.

\section{Introduction}

Discovered in 1966 [1], the hepatitis B virus (HBV) infection is a public health threat worldwide [2]. Globally, 240 million people are reported to be infected with HBV [3]. HBV infection led to progressive liver fibrosis and a significantly increased risk of liver cancer. About 650,000 people die from HBV-related cirrhosis or hepatocellular carcinoma every year [4]. The situation in China is more serious, with approximately 170 million HBV-infected people $[5,6]$. Therefore, in-depth exploration of pathological features and pathogenesis is of great significance for HBV control and prevention.

Nowadays, many regulators related to HBV have been studied, which are involved in the pathogenic process of HBV. Genome-wide association study (GWAS) has been used to identify genetic variants located in genes such as HLA-C [7], NOTCH4 [8], and TCF19 [9]. Moreover, the HBx protein enhanced the invasion and metastasis of liver cancer both in vivo and vitro [10], and the truncation of this protein can initiate hepatocarcinogenesis [11]. For the ther- 
apy of $\mathrm{HBV}$ infection, the inhibition of virus replication is one of the major approaches identified by the current researches and exhibits to reduce patient mortality and morbidity $[12,13]$. Notably, toll-like receptor (TLR) ligands can be used as one of the promising antiviral drug targets for $\mathrm{HBV}$ infection [14]. Specifically, the pathology of HBV disease is closely associated with chronic inflammation, which is a dynamic process orchestrated by the complex interplay between virus replication and host immune response [15]. And multiple key regulators were related to modulate HBV infection and inflammation, such as IFI16, AIM2, and p46 [15-18].

Over the past decades, several antiviral drug targets for HBV infection had been discovered $[19,20]$. For example, hnRNPK was identified to modulate the replicative efficiency of HBV [19]. Knockdown of hnRNPK resulted in a reduction of $\mathrm{HBV}$ viral load [19]. PLK1 is a key host factor for HBV replication in cells [20]. Blocking PLK1 could inhibit HBV DNA biosynthesis and strongly suppressed HBV infection in a mouse model [20]. However, current HBV treatment still cannot effectively eradicate the virus from chronic hepatitis $B$ patients [21, 22]. HBV treatment options include only nucleoside/nucleotide analogs (NUCs) and the immunomodulatory agent interferon-alpha (IFN- $\alpha$ ) [23, 24]. Moreover, the risk of $\mathrm{HBV}$ reactivation rises when patients receive immunosuppressive or antitumor therapy [25]. Therefore, the identification of drug targets and underlying mechanism for $\mathrm{HBV}$-infected patients is urgently needed. In this study, we collected transcriptome data from public database and conducted a systematic data analysis, aiming to identify genes involved in $\mathrm{HBV}$ infection and uncover the underlying mechanism.

\section{Materials and Methods}

2.1. Collection of Gene Expression Data of HBV Patients. The gene expression data was obtained from Gene Expression Omnibus (GEO) with accession GSE84044 [26], which included 124 chronic hepatitis B (CHB) patients. The clinical characteristics are summarized in Table 1. The count-based gene expression matrix was used for the analysis in this study.

2.2. Consensus Clustering Algorithm. The consensus clustering of samples from the GSE84044 dataset was conducted by the ConsensusClusterPlus $\mathrm{R}$ package [27]. The number of clusters was determined by the cumulative distribution function (CDF) and consistency score (greater than 0.8 in all clusters).

2.3. Liver Index Calculation. We performed the single-sample gene set enrichment analysis (ssGSEA) using the $\mathrm{R}$ gsva package [28] to calculate the liver index using 93 liverspecific genes, which represent the normal liver metabolism capability from previous study [29].

2.4. Differential Gene Expression Analysis. The differential expression analysis was conducted by R DESeq 2 package [30]. The genes with adjusted $p$ value $<0.05$ and $\log _{2}$ fold change $>1$ were considered as differentially expressed.
TABLE 1: The summarized clinical characteristics of the $124 \mathrm{CHB}$ patients.

\begin{tabular}{lcc}
\hline Clinical factor & \# of samples $(n=124)$ & Ratio \\
\hline Gender & 36 & \\
$\quad$ Female & 88 & $29.03 \%$ \\
$\quad$ Male & & $70.97 \%$ \\
Age & 92 & $74.19 \%$ \\
$\quad<50$ years & 32 & $25.81 \%$ \\
$>50$ years & & \\
Scheuer score grading & 37 & $29.84 \%$ \\
0 & 33 & $26.61 \%$ \\
1 & 34 & $27.42 \%$ \\
2 & 15 & $12.10 \%$ \\
3 & 5 & $4.03 \%$ \\
4 & & \\
Scheuer score staging & 43 & $34.68 \%$ \\
0 & 20 & $16.13 \%$ \\
1 & 33 & $26.61 \%$ \\
2 & 18 & $14.52 \%$ \\
3 & 10 & $8.06 \%$ \\
4 & &
\end{tabular}

2.5. Evaluation of Tumor-Infiltrating Immune Cells. The proportions of 22 tumor-infiltrating lymphocyte subsets in the liver tissues were calculated by CIBERSORT [31]. Besides, $p$ $<0.05$ was regarded as an accurate immune cell fraction, and the cell proportions between the groups were compared by the Wilcoxon-rank sum test.

2.6. Functional Enrichment Analysis. Gene ontology (GO) and Kyoto Encyclopedia of Genes and Genomes (KEGG) pathways were performed for each selected module by overrepresentation enrichment analysis using $\mathrm{R}$ clusterProfiler package [32]. Items with adjusted $p<0.05$ were regarded to be significant. The enrichment analysis of liver-specific genes was conducted by gene set enrichment analysis. The genes were ranked by the statistics calculated by R DESeq2 [30] package.

2.7. Statistical Analysis. Student's $t$-test was used to compare gene expression differences between tumor and normal tissues. All the statistics were done using the R software (version 4.0.2). $p$ value $<0.05$ was set as statistically significant for all the analyses.

\section{Results}

3.1. Identification of Molecular Subgroups in Patients with Hepatitis B Virus. Based on the gene expression data of 124 HBV patients (GSE84044), the consensus clustering algorithm was used to divide all samples into three categories, namely, C1, C2, and C3, with 38, 57, and 29 samples, respectively (Figure 1(a)). Samples were divided into three categories based on the cumulative distribution function (CDF) and consistency score. The CDF analysis showed that in the 

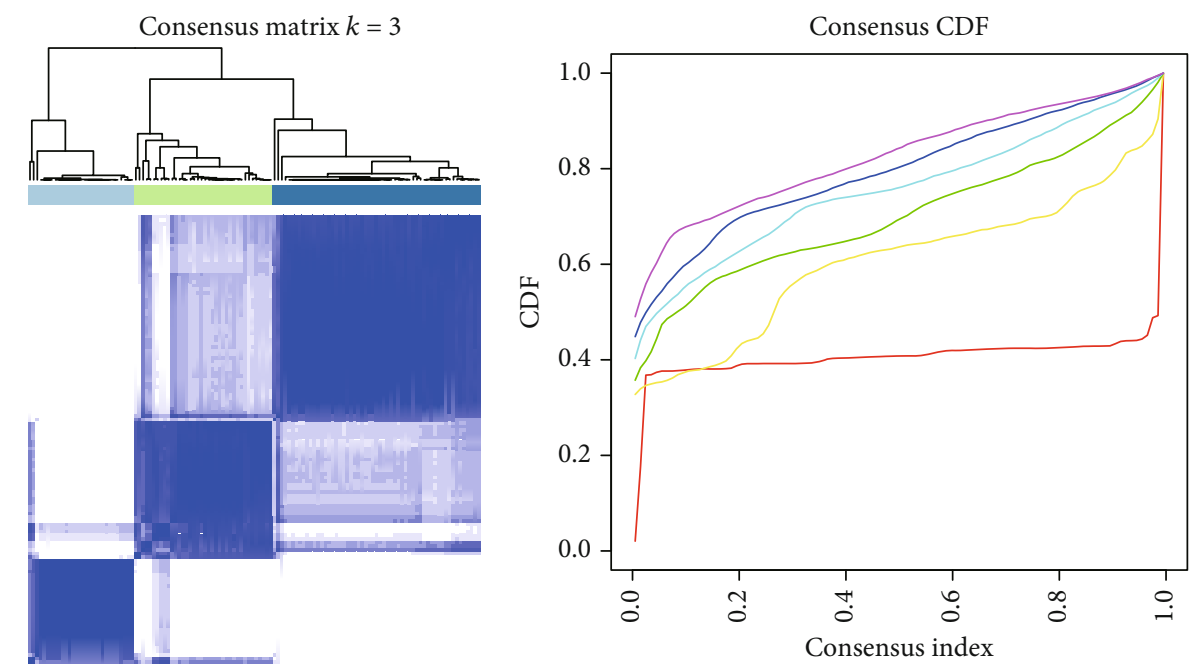

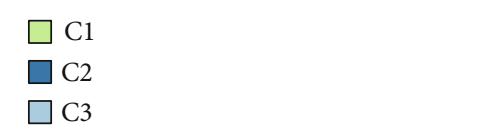

(a)

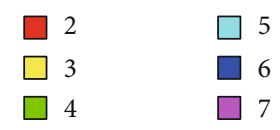

(b)

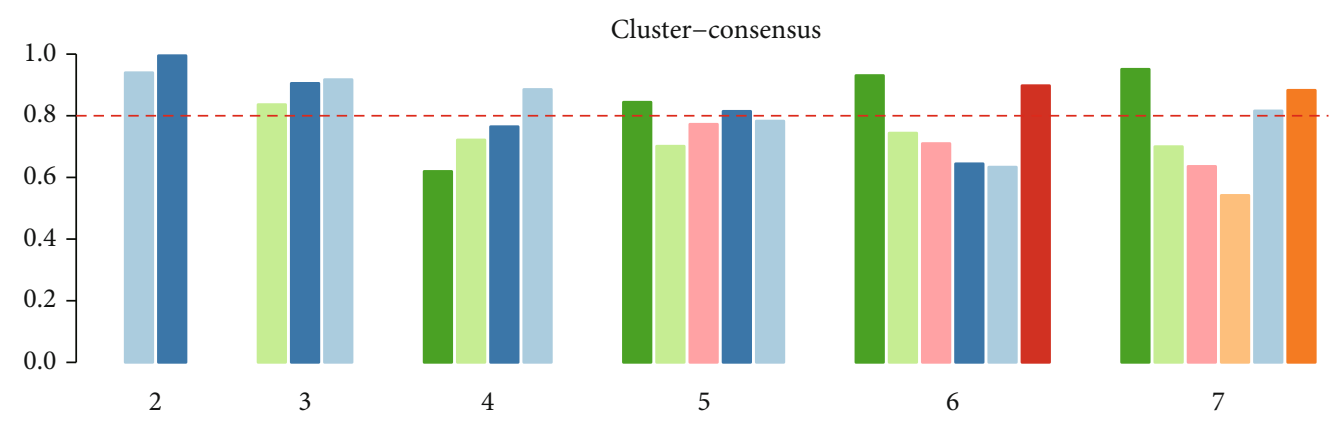

(c)

FIGURE 1: Identification of molecular subgroups in patients with hepatitis B virus. (a) $124 \mathrm{HBV}$ patients were used to divide all samples into three categories, namely, C1, C2, and C3, with 38, 57, and 29 samples, respectively. (b) The CDF analysis of the three categories. (c) The consistency score of each cluster was determined.

three categories, the area under the CDF curve did not increase significantly (Figure 1(b)); at the same time, the consistency score must be greater than $80 \%$ in each cluster (Figure 1(c)). Ultimately, three categories were selected for downstream analysis.

3.2. The Three Subgroups Have Significant Differences in Age, Fibrosis, and Inflammatory Levels. To further explore the clinical significance of the sample classification, the age, fibrosis, and inflammation of the three groups of samples were compared. Specifically, the age of the C2 group was younger than that of the $\mathrm{C} 1$ and $\mathrm{C} 3$ groups, but no difference was observed between the $\mathrm{C} 1$ and $\mathrm{C} 3$ groups (Figure 2(a)). The degree of fibrosis and verification was divided into 5 levels, from 0 to 4 points. The higher the score, the more serious the fibrosis. The proportions of the above five levels in the $\mathrm{C} 1, \mathrm{C} 2$, and $\mathrm{C} 3$ groups were significantly different (Figures 2(b) and 2(c)). Specifically, the degree of fibrosis and inflammation of the $\mathrm{C} 2$ group was lighter than that of the $\mathrm{C} 1$ and $\mathrm{C} 3$ groups, of which the $\mathrm{C} 3$ group was the most severe, followed by the $\mathrm{C} 1$ group. These results indicated that
C2 might have a higher grade in the pathogenetic process of HBV infection.

3.3. Molecular Characterization of the Molecular Subgroups. To further explore the differences in the molecular level of each group, the DESeq2 package was employed, and three groups were compared with each other. A total of 2006 differential genes were screened out $\left(\right.$ FDR $<0.1$ and $\log _{2}$ fold change $>0.5$, Supplementary Table S1). Unsupervised clustering of the above 2006 genes can classify these genes into four modules, named M1-M4 (Figure 3(a), Supplementary Table S2). Through gene set enrichment analysis, it was found that M1 was mainly enriched by metabolic pathways and PPAR signaling, which were mainly expressed in the $\mathrm{C} 1$ and $\mathrm{C} 2$ groups; M2 and M4 were mainly enriched by the inflammation-related pathways, and the specific pathway of M3 was proteoglycans in cancer, and M2, M3, and M4 were mainly expressed in the $\mathrm{C} 1$ and $\mathrm{C} 3$ groups. The high expression of the two groups (Figure $3(\mathrm{~b})$ ) indicated that these two 


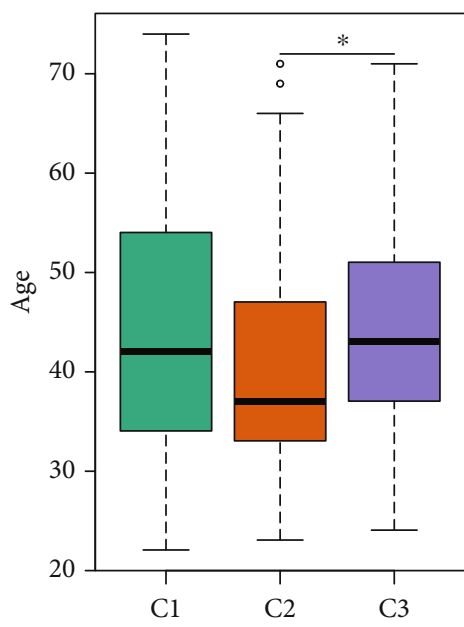

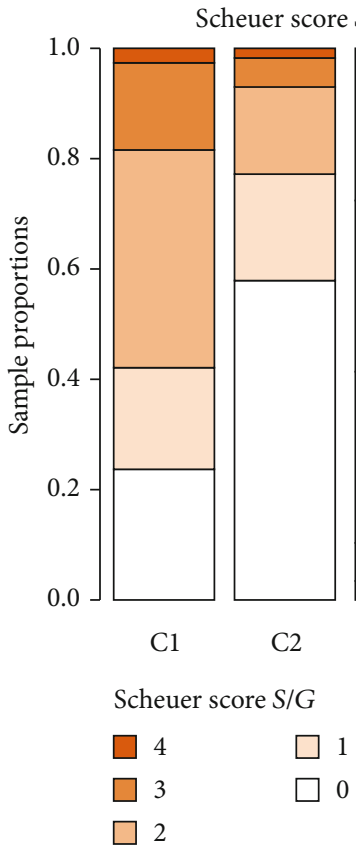

(b)

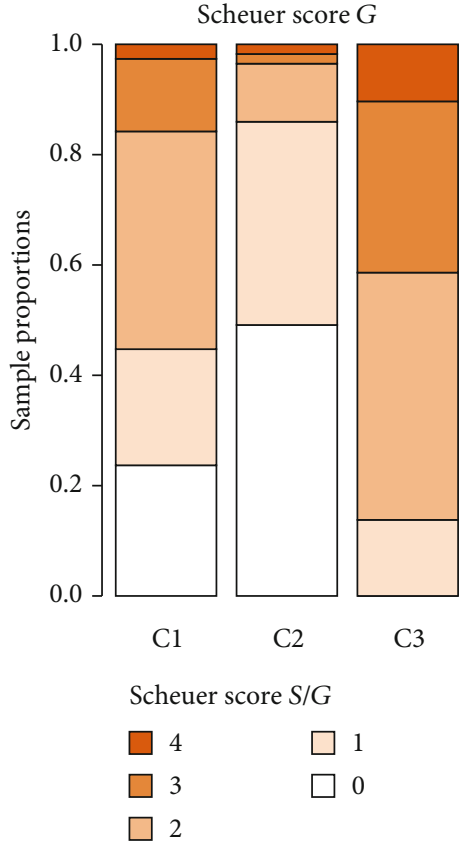

(c)

Figure 2: Significant differences in age, fibrosis, and inflammatory levels in three subgroups. (a) The distribution of age in C1, C2, and C3 groups was shown. (b, c) The proportions of fibrosis in $\mathrm{C} 1, \mathrm{C} 2$, and $\mathrm{C} 3$ groups were significantly different.

groups of patients had a higher tendency to precancerous lesions.

\subsection{Subgroup C2 Preserves a Higher Liver Functionality Than} C1 and C3. Since module M1 is mainly a metabolic pathway and is highly expressed in the C2 group, we compared C2 and $\mathrm{C} 1$ with $\mathrm{C} 3$ and found that the liver-specifically expressed genes [29] were highly enriched in C2 vs. C1 and C3 highly expressed gene (Figure $4(\mathrm{a}), \mathrm{FDR}<0.05)$. Combining the single-sample gene set enrichment analysis (ssGSEA) method and 93 liver-specifically expressed genes, a liver index (liver index) was constructed. Results showed that the liver index was significantly higher in the C2 group than in the $\mathrm{C} 1$ and $\mathrm{C} 3$ groups, and the liver index of the $\mathrm{C} 3$ group was the lowest (Figure 4(b)). At the same time, the liver index was also highly negatively correlated with fibrosis and inflammation levels (Figures 4(c) and 4(d)).

3.5. Differential Abundances of Immune Cells in the Molecular Subgroups. As the C3 group has a higher level of inflammation, the CIBERSORT was used to calculate the relative proportion of immune cells in each sample. Specifically, the macrophage M1/M2 and neutrophils showed significant difference between the three groups (Figure 5(a)). It is worth noting that M1 and M2 were highly negatively correlated (Figure 5(b)), indicating that these two cell types may be mutually exclusive. Specifically, M1 was mainly abundant in the $\mathrm{C} 3$ group, and M2 and neutrophils were mainly abundant in the C2 group (Figure 5(c)).

\section{Discussion}

More than 300 million people worldwide are infected with $\mathrm{HBV}$, with a higher infection rate in developing countries [33-35]. In particular, the incidence of $\mathrm{HBV}$ infection exceeds 8 percent in most Asian regions [33]. Besides, three-quarters of persons infected with $\mathrm{HBV}$ do not even know they are infected [33]. There was a significant difference in genotypes of infants between the HBV-infected pregnant women and those without HBV infection [36]. This causal relationship may be $\mathrm{HBV}$-driven [36]. Meanwhile, $\mathrm{HBV}$ is regarded as a human oncogenic virus, but the molecular mechanism of its tumorigenesis is unclear [37]. In this study, bioinformatics methods were used to analyze the gene expression data of $124 \mathrm{HBV}$ patients to further explore the different molecular subgroups of $\mathrm{HBV}$ patients, including age, fibrosis, inflammation degree, and related pathways. Furthermore, the liver index was calculated using 93 liverspecific genes. The liver-specific gene expression in different molecular subgroups of HBV patients was thoroughly analyzed and then compared with fibrosis and inflammation levels.

From the gene expression data of $124 \mathrm{HBV}$ patients, three types of submolecules were identified, namely, C1, C2, and C3. Results showed that the C2 group was the youngest and the C3 group had the most severe liver fibrosis and inflammation. The $\mathrm{C} 1$ and $\mathrm{C} 2$ groups were closely related to metabolic-related pathways, while inflammation and cancer-related pathways were closely related to the $\mathrm{C} 1$ and C3 groups. Many studies have shown that some pathways 


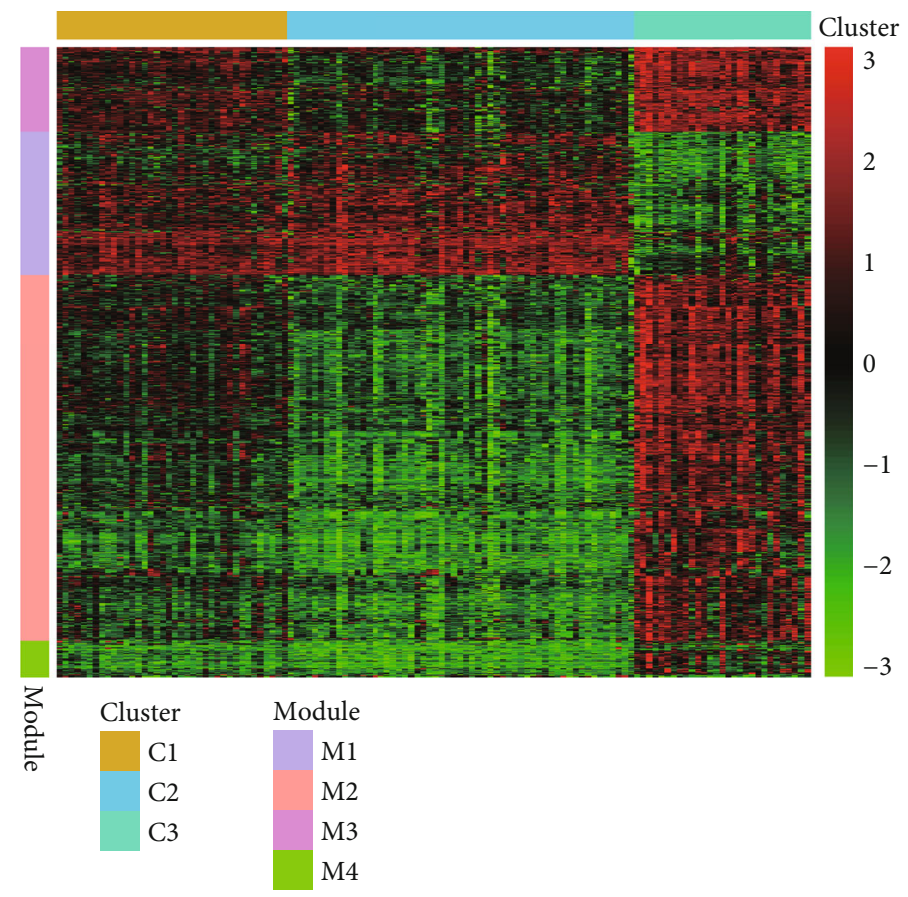

(a)

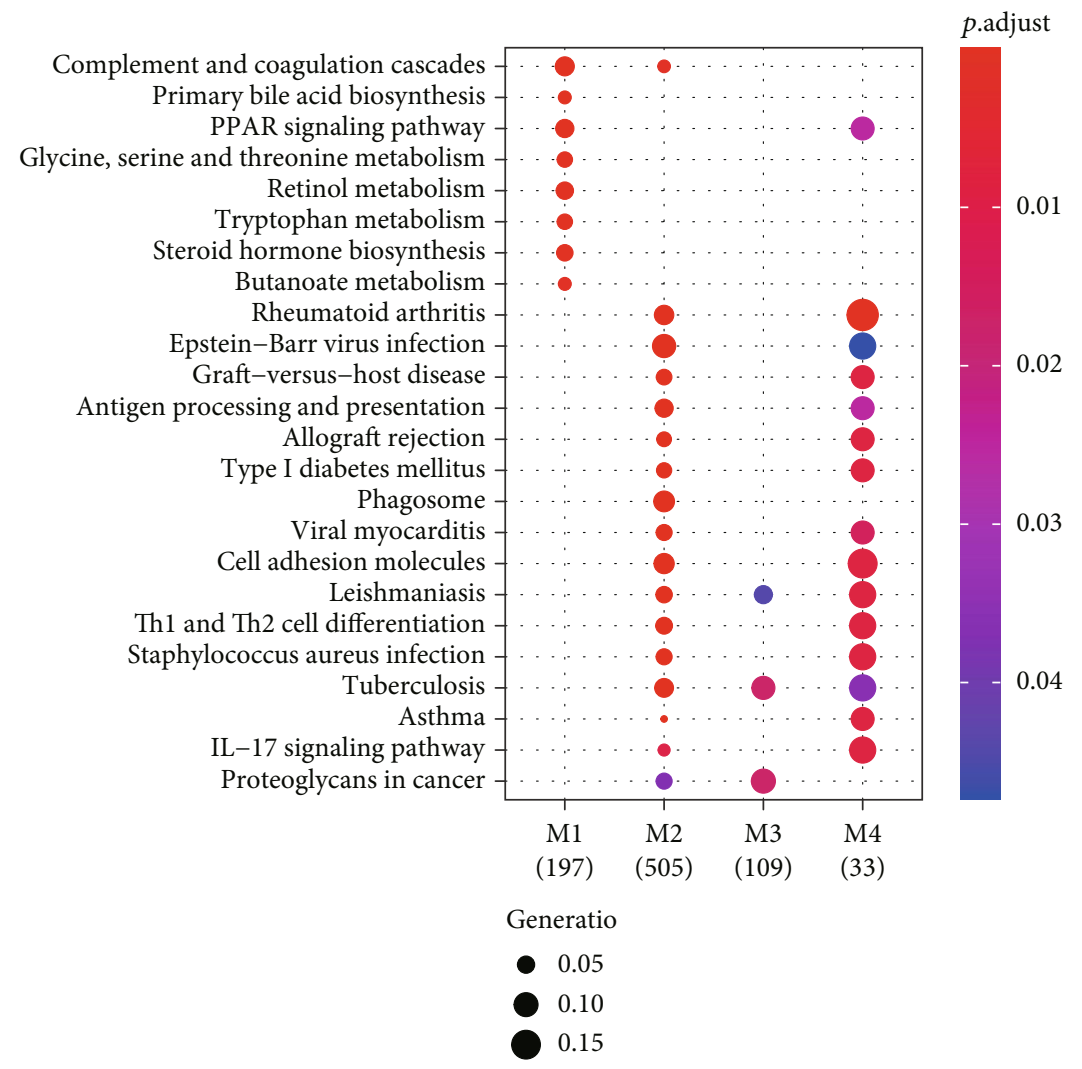

(b)

FIGURE 3: Molecular characterization of the molecular subgroups. (a) Unsupervised clustering of the above 2006 genes can classify these genes into four modules, named M1-M4. (b) Bioinformatics analysis of differently expressed genes in M1-M4 is shown.

in HBV patients are different from those in normal people, which is also a starting point for HBV treatment. For example, the HBV-STAT3-miR-328-3p-FOXO4 axis participates in the chronic HBV infection pathogenesis [38]. HBV impairs IFN activity by hijacking the IFN/JAK/STAT pathway through $\mathrm{HBeAg}$ [39]. The liver index of the C2 group 


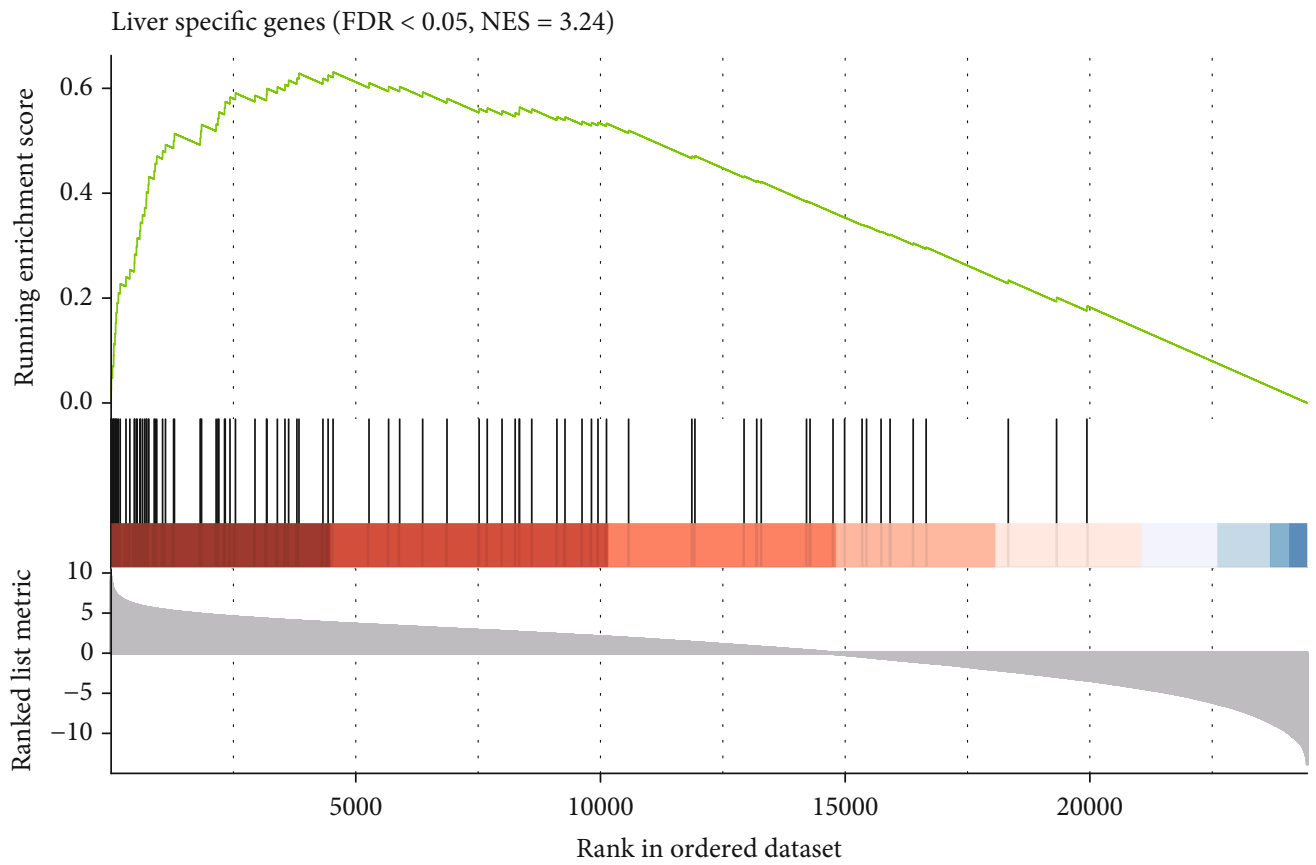

(a)

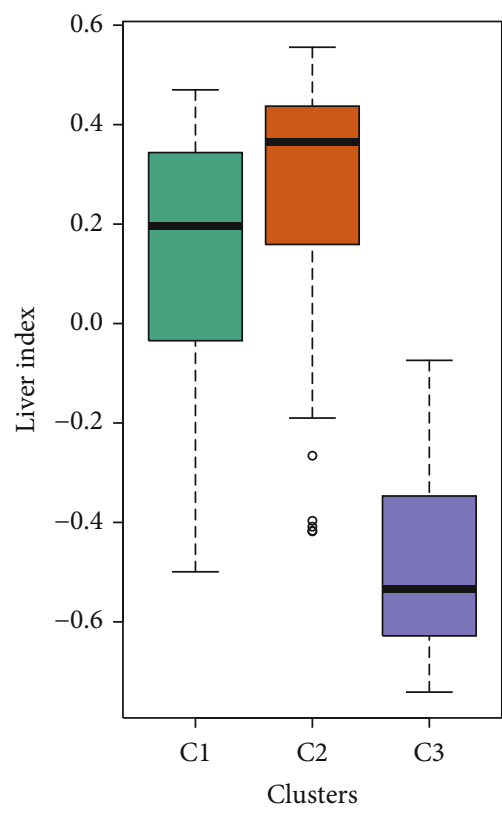

(b)

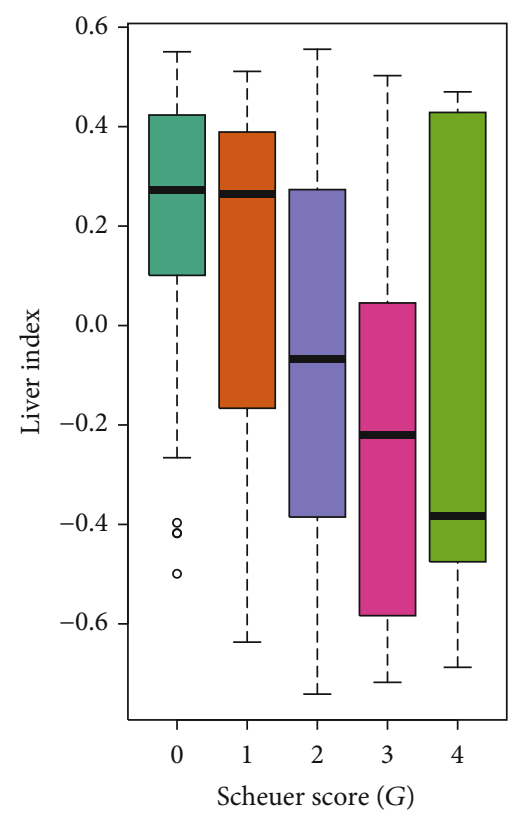

(c)

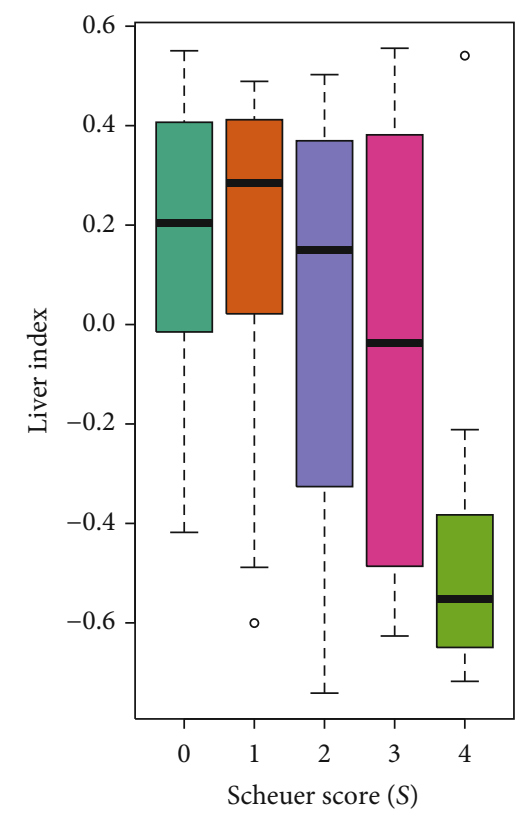

(d)

Figure 4: Subgroup C2 preserves a higher liver functionality than C1 and C3. (a) The liver-specifically expressed genes were highly enriched in $\mathrm{C} 2$ vs. $\mathrm{C} 1$ and $\mathrm{C} 3$ highly expressed gene. (b) Liver index was significantly higher in the $\mathrm{C} 2$ group than in the $\mathrm{C} 1$ and $\mathrm{C} 3$ groups, and the liver index of the C3 group was the lowest. Liver index was also highly negatively correlated with (c) fibrosis and (d) inflammation levels.

was higher than that of the other two groups, and the liver index of the C3 group was the lowest. At the same time, the liver index was highly negatively correlated with fibrosis and inflammation, which was consistent with the enrichment pathway analysis.

Of note, we identified 2006 differentially expressed genes among the three types of HBV patients. Using unsupervised clustering analysis, we revealed 2006 genes could be classified into four modules, named M1-M4. Bioinformatics analysis indicated these modules were related to regulate multiple crucial pathways in HBV infection and liver tumorigenesis. For example, M1 was enriched in metabolic pathways and PPAR signaling. Peroxisome proliferator-activated receptor-gamma coactivator 1 alpha (PGC-1alpha), a major metabolic regulator, was identified to strongly coactivate HBV transcription [40, 41]. M3 was especially involved in 

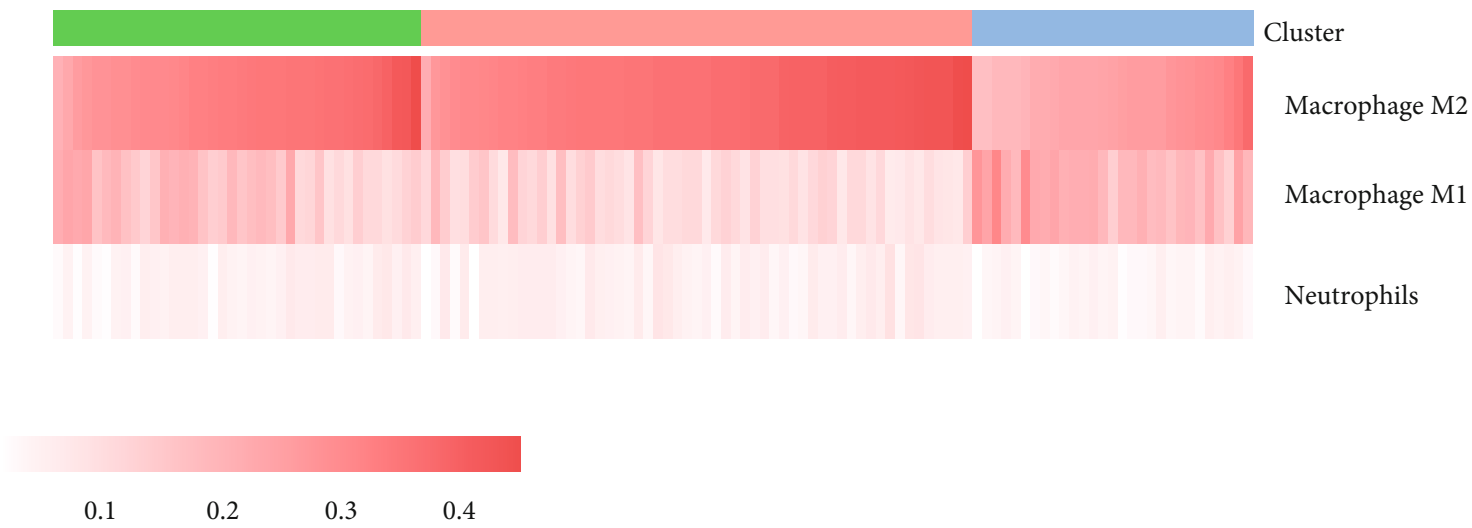

Cluster
C1
C2
C3

(a)

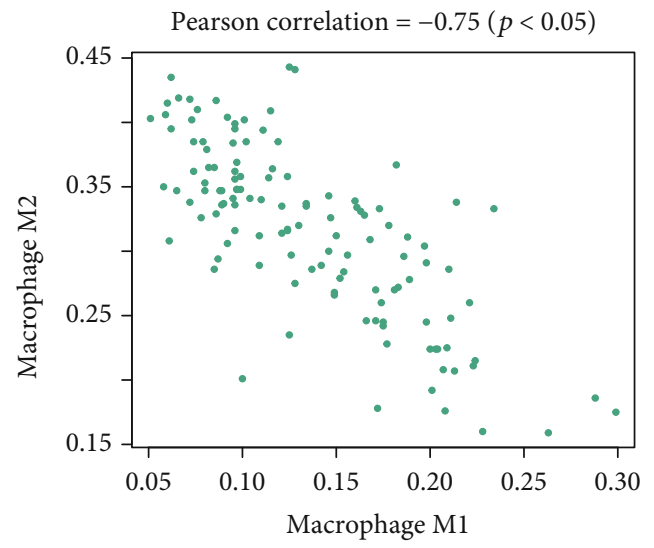

(b)
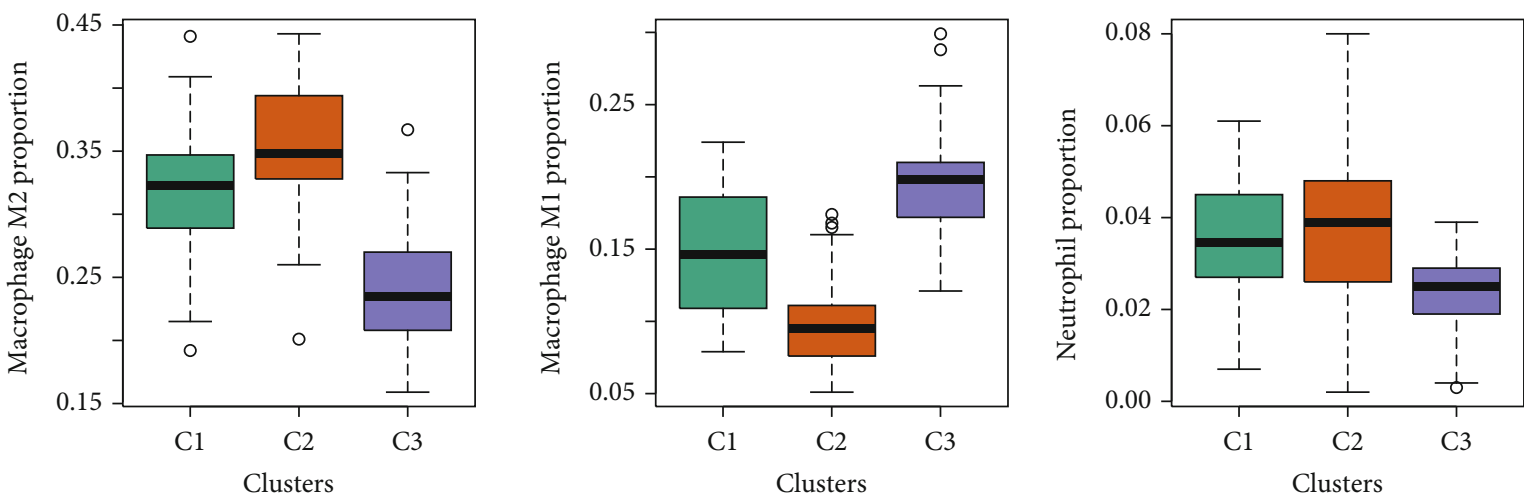

(c)

FiguRE 5: Differential abundances of immune cells in the molecular subgroups. (a) The macrophage M1/M2 and neutrophils showed significant difference between the three groups. (b) M1 and M2 were highly negatively correlated. (c) The levels of macrophage M2, macrophage $\mathrm{M} 1$, and neutrophils in $\mathrm{C} 1, \mathrm{C} 2$, and $\mathrm{C} 3$ groups are shown.

regulating proteoglycans in cancer. Proteoglycans are characterized by a central protein backbone that is decorated with linear sulfated glycosaminoglycan side chains. Proteoglycans were related to regulate the biochemical and mechanical properties of the interstitial extracellular matrix. Various studies demonstrated proteoglycans enhanced malignant transformation and alter antitumor therapy response [42]. M2 and M4 were mainly enriched by the inflammation- 
related pathways, such as IL-17 signaling and Th1 and Th2 cell differentiation, which was involved in modulating both HBV infection and liver cancer progression. For example, IL-17 activates the IL-6/STAT3 signal pathway in the proliferation of hepatitis B virus-related hepatocellular carcinoma [43]. Hepatitis B virus induces IL-23 production in antigenpresenting cells and causes liver damage via the IL-23/IL-17 axis [44].

Several limitations should also be noted in this study. Firstly, the conclusion of this study was obtained using bioinformatics analysis and lacking of experimental validation. We will validate the clinical significance of the sample classification by collecting clinical samples. Secondly, several hub signaling pathways were revealed to modulate HBV infection, such as PPAR signaling, and the inflammation-related pathways. Exploring the effects of this signaling on HBV infection with pathway-specific inhibitors could further strengthen the findings of this study. In summary, our study for the first time comprehensively demonstrated the potential mechanisms of $\mathrm{HBV}$ promoting liver fibrosis and tumor progression.

\section{Conclusions}

In conclusion, using bioinformatics to complete and analyze the gene expression data of HBV patients, a lot of useful information is obtained, which provides a reference for further understanding of the pathogenic mechanism of $\mathrm{HBV}$ and has predictive value.

\section{Data Availability}

Previously reported gene expression and clinical data were used to support this study and are available at Gene Expression Omnibus (GEO, https://www.ncbi.nlm.nih.gov/gds). These prior studies (and datasets) are cited at relevant places within the text as references.

\section{Conflicts of Interest}

The authors declare that they have no conflicts of interest.

\section{Authors' Contributions}

X. L. and H. S. designed this study. C. Z. and J. L. conducted the data analysis and visualization and experiments. C. Z., L. Y., F. X., and J. L. contributed to the writing of the paper and setting of figures. Conghui Zhang and Jie $\mathrm{Li}$ contributed equally to this work.

\section{Acknowledgments}

This work was supported by the Open Research Program of the State Key Laboratory of Virology of China (Grant No. 2019KF004), the Outstanding Leaders Training Program of Pudong Health Bureau of Shanghai (Grant No. PWR1201805), the Key Disciplines Group Construction Project of Pudong Health Bureau of Shanghai (Grant No. PWZxq2017-15), and the Open Funds of Key Laboratory of
Diagnosis and Treatment of Digestive System Tumors of Zhejiang Province (Grant No. KFJJ-202007).

\section{Supplementary Materials}

Supplementary 1. Supplementary Table S1: the subgroupspecific genes in the three subgroups of $\mathrm{CHB}$ patients.

Supplementary 2. Supplementary Table S2: the functional module-specific genes in $\mathrm{CHB}$ patients.

\section{References}

[1] H. J. Alter and B. S. Blumberg, "Further studies on a "new" human isoprecipitin system (Australia antigen)," Blood, vol. 27, no. 3, pp. 297-309, 1966.

[2] Y. Shi and M. Zheng, "Hepatitis B virus persistence and reactivation," BMJ, vol. 370, p. m2200, 2020.

[3] J. H. MacLachlan and B. C. Cowie, "Hepatitis B virus epidemiology," Cold Spring Harbor Perspectives in Medicine, vol. 5, no. 5, p. a021410, 2015.

[4] M. J. Tong, L. M. Blatt, K. B. Tyson, and V. W. Kao, "Death from liver disease and development of hepatocellular carcinoma in patients with chronic hepatitis B virus infection: a prospective study," Gastroenterology \& hepatology, vol. 2, no. 1, pp. 41-47, 2006.

[5] Y. P. Yan, H. X. Su, Z. H. Ji, Z. J. Shao, and Z. S. Pu, "Epidemiology of hepatitis B virus infection in China: current status and challenges," Journal of Clinical and Translational Hepatology, vol. 2, no. 1, pp. 15-22, 2014.

[6] D. Li, Y. Long, T. Wang et al., "Epidemiology of hepatitis C virus infection in highly endemic HBV areas in China," PLoS One, vol. 8, no. 1, article e54815, 2013.

[7] Z. Hu, Y. Liu, X. Zhai et al., "New loci associated with chronic hepatitis B virus infection in Han Chinese," Nature Genetics, vol. 45, no. 12, pp. 1499-1503, 2013.

[8] D. K. Jiang, X. P. Ma, H. Yu et al., "Genetic variants in five novel loci including CFB and CD40 predispose to chronic hepatitis B," Hepatology, vol. 62, no. 1, pp. 118-128, 2015.

[9] Y. J. Kim, H. Y. Kim, J. H. Lee et al., "A genome-wide association study identified new variants associated with the risk of chronic hepatitis B," Human Molecular Genetics, vol. 22, no. 20, pp. 4233-4238, 2013.

[10] S. Yang, Y. Liu, X. Feng et al., "HBx acts as an oncogene and promotes the invasion and metastasis of hepatocellular carcinoma both in vivo and vitro," Digestive and Liver Disease, vol. 53, pp. 360-366, 2021.

[11] Y. Zhang, Q. Yan, L. Gong et al., "C-terminal truncated HBx initiates hepatocarcinogenesis by downregulating TXNIP and reprogramming glucose metabolism," Oncogene, vol. 40, no. 6, pp. 1147-1161, 2020.

[12] M. Ringelhan, J. A. McKeating, and U. Protzer, "Viral hepatitis and liver cancer," Philosophical Transactions of the Royal Society of London. Series B, Biological Sciences, vol. 372, no. 1732, 2017.

[13] G. Dusheiko, "Towards the elimination and eradication of hepatitis B," Journal of Virus Eradication, vol. 1, no. 1, pp. 412, 2015.

[14] K. Du, J. Liu, R. Broering et al., "Recent advances in the discovery and development of TLR ligands as novel therapeutics for chronic HBV and HIV infections," Expert opinion on drug discovery, vol. 13, no. 7, pp. 661-670, 2018. 
[15] X. Pang, X. Li, Z. Mo et al., "IFI16 is involved in HBVassociated acute-on-chronic liver failure inflammation," BMC Gastroenterology, vol. 18, no. 1, p. 61, 2018.

[16] H. Chen, G. He, Y. Chen, X. Zhang, and S. Wu, "Differential activation of NLRP3, AIM2, and IFI16 inflammasomes in humans with acute and chronic hepatitis B," Viral Immunology, vol. 31, no. 9, pp. 639-645, 2018.

[17] Q. Yan, M. Li, Q. Liu et al., "Molecular characterization of woodchuck IFI16 and AIM2 and their expression in woodchucks infected with woodchuck hepatitis virus (WHV)," Scientific Reports, vol. 6, no. 1, p. 28776, 2016.

[18] W. Li, Y. Jiang, X. Wang et al., "Natural killer p46 controls hepatitis B virus replication and modulates liver inflammation," PLoS One, vol. 10, no. 8, article e0135874, 2015.

[19] L. F. Ng, M. Chan, S. H. Chan et al., "Host heterogeneous ribonucleoprotein $\mathrm{K}$ (hnRNP K) as a potential target to suppress hepatitis B virus replication," PLoS Medicine, vol. 2, no. 7, article e163, 2005.

[20] A. Diab, A. Foca, F. Fusil et al., "Polo-like-kinase 1 is a proviral host factor for hepatitis B virus replication," Hepatology, vol. 66, no. 6, pp. 1750-1765, 2017.

[21] R. F. Edlich, M. L. Martin, A. O. Diallo, and L. Buchanan, "Hepatitis B virus: a comprehensive strategy for eliminating transmission in the United States," Journal of Long-Term Effects of Medical Implants, vol. 13, no. 2, pp. 117-126, 2003.

[22] F. X. Bosch, T. R. Broker, D. Forman et al., "Comprehensive control of human papillomavirus infections and related diseases," Vaccine, vol. 31, Suppl 7, pp. H1-31, 2013.

[23] S. Tavakolpour, M. Darvishi, H. S. Mirsafaei, and M. Ghasemiadl, "Nucleoside/nucleotide analogues in the treatment of chronic hepatitis B infection during pregnancy: a systematic review," Infectious Diseases, vol. 50, no. 2, pp. 95-106, 2018.

[24] J. Fung, C. L. Lai, W. K. Seto, and M. F. Yuen, "Nucleoside/nucleotide analogues in the treatment of chronic hepatitis B," The Journal of Antimicrobial Chemotherapy, vol. 66, no. 12, pp. 2715-2725, 2011.

[25] R. Loomba and T. J. Liang, "Hepatitis B reactivation associated with immune suppressive and biological modifier therapies: current concepts, management strategies, and future directions," Gastroenterology, vol. 152, no. 6, pp. 1297-1309, 2017.

[26] M. Wang, Q. Gong, J. Zhang et al., "Characterization of gene expression profiles in HBV-related liver fibrosis patients and identification of ITGBL1 as a key regulator of fibrogenesis," Scientific Reports, vol. 7, no. 1, p. 43446, 2017.

[27] M. D. Wilkerson and D. N. Hayes, "ConsensusClusterPlus: a class discovery tool with confidence assessments and item tracking," Bioinformatics, vol. 26, no. 12, pp. 1572-1573, 2010.

[28] M. S. Rooney, S. A. Shukla, C. J. Wu, G. Getz, and N. Hacohen, "Molecular and genetic properties of tumors associated with local immune cytolytic activity," Cell, vol. 160, no. 1-2, pp. 48-61, 2015.

[29] D. S. Kim, J. W. Ryu, M. Y. Son et al., "A liver-specific gene expression panel predicts the differentiation status of in vitro hepatocyte models," Hepatology, vol. 66, no. 5, pp. 16621674, 2017.

[30] M. I. Love, W. Huber, and S. Anders, "Moderated estimation of fold change and dispersion for RNA-seq data with DESeq2," Genome Biology, vol. 15, no. 12, p. 550, 2014.

[31] B. Chen, M. S. Khodadoust, C. L. Liu, A. M. Newman, and A. A. Alizadeh, "Profiling tumor infiltrating immune cells with
CIBERSORT," Methods in Molecular Biology, vol. 1711, pp. 243-259, 2018.

[32] G. Yu, L. G. Wang, Y. Han, and Q. Y. He, “clusterProfiler: an R package for comparing biological themes among gene clusters," OMICS, vol. 16, no. 5, pp. 284-287, 2012.

[33] N. P. Nelson, P. J. Easterbrook, and B. J. McMahon, "Epidemiology of hepatitis B virus infection and impact of vaccination on disease," Clinics in Liver Disease, vol. 20, no. 4, pp. 607628, 2016.

[34] H. Saffar, A. Ajami, M. J. Saffar et al., "Prevalence of hepatitis B virus seromarkers in young adults vaccinated at birth; impact on the epidemiology of hepatitis B infection in Iran," Hepatitis Monthly, vol. 14, no. 5, article e17263, 2014.

[35] I. M. Jacobson, J. L. Dienstag, B. G. Werner, D. B. Brettler, P. H. Levine, and I. K. Mushahwar, "Epidemiology and clinical impact of hepatitis D virus (delta) infection," Hepatology, vol. 5, no. 2, pp. 188-191, 1985.

[36] S. Polywka, H. Feucht, B. Zöllner, and R. Laufs, "Hepatitis C virus infection in pregnancy and the risk of mother-to-child transmission," European Journal of Clinical Microbiology \& Infectious Diseases, vol. 16, no. 2, pp. 121-124, 1997.

[37] U. N. Mui, C. T. Haley, and S. K. Tyring, "Viral oncology: molecular biology and pathogenesis," Journal of Clinical Medicine, vol. 6, no. 12, 2017.

[38] X. Fu, Y. Ouyang, J. Mo, R. Li, L. Fu, and S. Peng, "Upregulation of microRNA-328-3p by hepatitis B virus contributes to THLE-2 cell injury by downregulating FOXO4," Journal of Translational Medicine, vol. 18, no. 1, p. 143, 2020.

[39] Y. Yu, P. Wan, Y. Cao et al., "Hepatitis B virus e antigen activates the suppressor of cytokine signaling 2 to repress interferon action," Scientific Reports, vol. 7, no. 1, p. 1729, 2017.

[40] L. Du, Y. Ma, M. Liu, L. Yan, and H. Tang, "Peroxisome proliferators activated receptor (PPAR) agonists activate hepatitis B virus replication in vivo," Virology Journal, vol. 14, no. 1, p. 96, 2017.

[41] C. R. Ondracek and A. McLachlan, "Role of peroxisome proliferator-activated receptor gamma coactivator lalpha in AKT/PKB-mediated inhibition of hepatitis B virus biosynthesis," Journal of Virology, vol. 85, no. 22, pp. 11891-11900, 2011.

[42] A. Barkovskaya, A. Buffone Jr., M. Žídek, and V. M. Weaver, "Proteoglycans as mediators of cancer tissue mechanics," Frontiers in Cell and Development Biology, vol. 8, p. 569377, 2020.

[43] L. Cong and L. Hu, "The value of the combination of hemoglobin, albumin, lymphocyte and platelet in predicting platinumbased chemoradiotherapy response in male patients with esophageal squamous cell carcinoma," International Immunopharmacology, vol. 46, pp. 75-79, 2017.

[44] Q. Wang, J. Zhou, B. Zhang et al., "Hepatitis B virus induces IL-23 production in antigen presenting cells and causes liver damage via the IL-23/IL-17 axis," PLoS Pathogens, vol. 9, no. 6, article e1003410, 2013. 